\title{
Antioxidant effects and mechanism of thiopental and propofol on the rabbit abdominal aortic endothelial dependent vasorelaxation against reactive oxygen species
}

\author{
In Kyu Kim ${ }^{1}$, Jung Kook Suh², and Ji Hyun Kim ${ }^{1}$ \\ Department of Anesthesiology and Pain Medicine, 'Samsung Changwon Hospital, Sungkyunkwan University School of Medicine, \\ Changwon, ${ }^{2}$ Hanyang University, Seoul, Korea
}

Reactive oxygen species (ROS), which are also generated in a normal condition in certain quantities, are generated in large quantities by ischemia or by oxygen resupply at the time of reperfusion and not reduced by the normal antioxidant systems of the body, eventually causing reperfusion injury in tissues [1].

In this study, we investigated whether thiopental and propofol, which are intravenous anesthetics, decrease endothelium injury by ROS and evaluated the mechanism for the decrease by causing endothelium-dependent relaxation of rabbit abdominal aortas.

All the experiments in this study were done after obtaining the approval of the Institutional Animal Care and Use Committee. Twenty four male New Zealand white rabbits (KOATECH, Pyeongtaek, Korea) weighing 2.0-2.5 kg were anesthetized with 3-5 vol\% sevoflurane and $100 \%$ oxygen $4 \mathrm{~L} / \mathrm{min}$. Heparin 600 $\mathrm{IU} / \mathrm{kg}$ was intravenously injected through the auricular marginal vein. Ten minutes after the heparin injection, the rabbit carotid artery was sectioned for exsanguinations, and the infra renal abdominal aorta was obtained.

ROS was generated by performing electrolysis (EL) of a $\mathrm{K}-\mathrm{H}$ solution in an experimental bath. Two circular Platinum wire electrodes $(7 \mathrm{~mm})$ were positioned at the lower part of the experimental bath. Hemangioendothelial injury by ROS was induced by exposure to $15 \mathrm{~mA} \mathrm{DC}$ constant current for $35 \mathrm{sec}-$ onds (EL+) [2,3].

Thiopental and propofol, intravenous anesthetics, were put into the experimental bath at concentrations of $10^{-5} \mathrm{M}, 3 \times 10^{-5} \mathrm{M}$,
$10^{-4} \mathrm{M}$, and $3 \times 10^{-4} \mathrm{M}$ and at concentrations of $10^{-4} \mathrm{M}, 3 \times 10^{-4} \mathrm{M}$, $10^{-3} \mathrm{M}$, and $3 \times 10^{-3} \mathrm{M}$, respectively. After 15 minutes of pretreatment, EL+ was performed for 35 seconds. The used K-H solution was replaced with new K-H solution. After contracting the abdominal aorta by putting in $\mathrm{NE}, \mathrm{ACh}$ at concentrations of $3 \times 10^{-8} \mathrm{M}, 10^{-7} \mathrm{M}, 3 \times 10^{-7} \mathrm{M}$, and $10^{-6} \mathrm{M}$ was continuously added to the bath to relax the annular slices.

$\mathrm{Cu} / \mathrm{Zn}$ superoxide dismutase (SOD) and catalase, which are important antioxidative enzymes, were pretreated with $0.8 \mathrm{mM}$ diethyldithiocarbamate (DETCA) [4], a SOD inhibitor, for 30 minutes and with $50 \mathrm{mM}$ 3-amino-1,2,4-triazole (3AT) [5], a catalase inhibitor, for 60 minutes.

While pretreating with DETCA and 3AT for 30 and 60 minutes in the experimental bath, respectively, thiopental $\left(3 \times 10^{-4} \mathrm{M}\right)$ or propofol $\left(3 \times 10^{-3} \mathrm{M}\right)$ was, respectively, added to the experimental bath 15 minutes before the completion of the pretreatment. After 15 minutes, EL was performed to generate ROS, and then the K-H solution was replaced with a new K-H solution. The annular slices were contracted and relaxed by adding NE and Ach to the bath.

All the data were expressed as the "Mean \pm Standard Error" and the degree of ACh relaxation, calculated in percentage, was used as the control value.

The comparison of the results between the pretreated groups with respect to the thiopental and propofol concentrations was done by a one-way ANOVA and Dunnett test was done for a post-hoc test. An unpaired t-test was done to compare the aortic

Corresponding author: Jung Kook Suh, M.D., Department of Anesthesiology and Pain Medicine, Hanyang University, 17, Haengdang-dong, Sungdong-gu, Seoul 133-792, Korea. Tel: 82-2-2290-8689, Fax: 82-2-2299-0742, E-mail: jksuh@hanyang.ac.kr

(c) This is an open-access article distributed under the terms of the Creative Commons Attribution Non-Commercial License (http:// creativecommons.org/licenses/by-nc/3.0/), which permits unrestricted non-commercial use, distribution, and reproduction in any medium, provided the original work is properly cited. 
tension between the DETCA or 3AT pretreated groups and nonpretreated group. Values having a $\mathrm{P}$ value less than 0.05 were considered as significant.

In the thiopental $10^{-5} \mathrm{M}, 3 \times 10^{-5} \mathrm{M}, 10^{-4} \mathrm{M}$, and $3 \times 10^{-4} \mathrm{M}$ pretreated groups, the relaxation reaction of vascular endothelium by ROS was proportional to the concentration. The rate of relaxation by $10^{-6} \mathrm{M}$ ACh was $-2.7 \pm 0.9,-13.3 \pm 2.5,-76.6 \pm$ 1.5 , and $-85.6 \pm 1.2 \%$, respectively. A comparison of the thiopental $10^{-5} \mathrm{M}$ group with the thiopental $3 \times 10^{-5} \mathrm{M}, 10^{-4} \mathrm{M}$, and $3 \times 10^{-4} \mathrm{M}$ groups showed that the relaxation rate of the annular slices was significantly higher in the thiopental $3 \times 10^{-5} \mathrm{M}, 10^{-4} \mathrm{M}$, and $3 \times 10^{-4} \mathrm{M}$ groups $(\mathrm{P}<0.001)$. Similar to the thiopental pretreated groups, the propofol $10^{-4} \mathrm{M}(\mathrm{n}=15), 3 \times 10^{-4} \mathrm{M}(\mathrm{n}=$ $15), 10^{-3} \mathrm{M}(\mathrm{n}=15)$, and $3 \times 10^{-3} \mathrm{M}(\mathrm{n}=15)$ groups showed a rate of relaxation by $10^{-6} \mathrm{M}$ ACh of $-4.6 \pm-1.5,-19.1 \pm 4.2$, $-61.6 \pm 3.3$, and $-83.1 \pm 1.7 \%$, respectively. A comparison of the propofol $10^{-4} \mathrm{M}$ group with the propofol $3 \times 10^{-4} \mathrm{M}, 10^{-3} \mathrm{M}$, and $3 \times 10^{-3} \mathrm{M}$ groups showed that the relaxation rate of the annular slices was significantly higher in the $3 \times 10^{-4} \mathrm{M}, 10^{-3} \mathrm{M}$, and $3 \times 10^{-3} \mathrm{M}$ groups $(\mathrm{P}<0.001)$.

The annular slices relaxation rate of the DETCA + thiopental $\left(3 \times 10^{-4} \mathrm{M}, \mathrm{n}=13, \mathrm{EL}+\right)$ group after ROS exposure was -65.8 $\pm 2.9 \%$ which was significantly lower than the control value (EL-) $-85.6 \pm 1.2 \%$ for the thiopental $3 \times 10^{-4} \mathrm{M}$ group $(\mathrm{P}<0.001)$. However, the annular slices relaxation rate of the $3 \mathrm{AT}+$ thiopental $\left(3 \times 10^{-3} \mathrm{M}, \mathrm{EL}+\right)$ group was $-87.5 \pm 1.3 \%$ which was not significantly different from the control value $-86.6 \pm 1.4 \%$ as well as the value of the thiopental $3 \times 10^{-4} \mathrm{M}$ group. The annular slices relaxation rate of DETCA + propofol $\left(3 \times 10^{-3} \mathrm{M}, \mathrm{n}\right.$ $=15, \mathrm{EL}+$ ) after ROX exposure by EL was $-51.6 \pm 2.3 \%$ which was significantly lower than the control value (EL-) $-85.1 \pm$

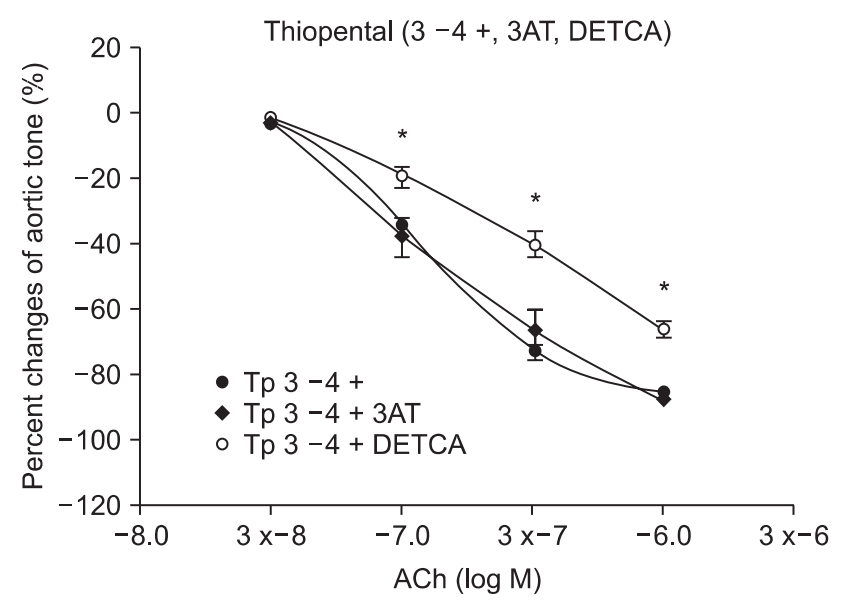

Fig. 1A. Concentration-response curves of acetylcholine (ACh)-induced endothelium-dependent relaxation after electrolysis in the in the presence of 3-amino-1,2,4-triazole (3AT), diethyldithiocarbamate (DETCA). Values are presented as mean \pm SEM. *A P value of less than 0.001 significantly different from control group (Thiopental $[\mathrm{Tp}] 3 \times 10^{-4} \mathrm{M}$ ).
$0.6 \%$ as well as the value $-85.5 \pm 1.8 \%$ for the propofol $3 \times$ $10^{-3} \mathrm{M}$ group $(\mathrm{P}<0.001)$ (Fig. $\left.1 \mathrm{~A}\right)$. However, the annular slices relaxation rate of the $3 \mathrm{AT}+$ propofol $\left(3 \times 10^{-3} \mathrm{M}, \mathrm{EL}+\right)$ group was $-88.8 \pm 0.8 \%$ which was not significantly different from the control value $-83.1 \pm 1.7 \%$ as well as the value of the propofol 3 $\times 10^{-3} \mathrm{M}$ group (Fig. 1B).

Because a superoxide anion radical is generated by the EL+ of $\mathrm{K}-\mathrm{H}$ solution, hydrogen peroxide is generated subsequently. Then, hydroxyl radicals are generated through the Harber Weiss and Fenton reactions. The decrease in the relaxation rate in both the thiopental and propofol groups pretreated with DETCA inhibiting SOD suggests that both thiopental and propofol have an effect similar to that of SOD. In both the thiopental and propofol groups pretreated with 3-AT, a catalase inhibitor, the enzyme eliminating hydrogen peroxide as ROS superoxide, which is the precursor of hydrogen peroxide, might have already been eliminated by the SOD-like effect, and thus, hydrogen peroxide might have not been generated. Therefore, the 3-AT pretreatment might be insignificant.

On the other hand, when hydrogen peroxide was put added to SH-SY5Y cells to cause cell injury following eight hours of propofol pretreatment, the effect of hydrogen peroxide was attenuated by the ERK pathway. According to the result, a long duration of propofol pretreatment may be required to prevent, through propofol, the injury caused by hydrogen peroxide.

Thiopental and propofol decreased the injury caused by ROS to the rabbit abdominal aorta endothelium. The mechanism for the antioxidant effect of thiopental and propofol did not show a similar effect to that of catalase, which is a hydrogen peroxide scavenger, but showed a superoxide anion scavenging effect.

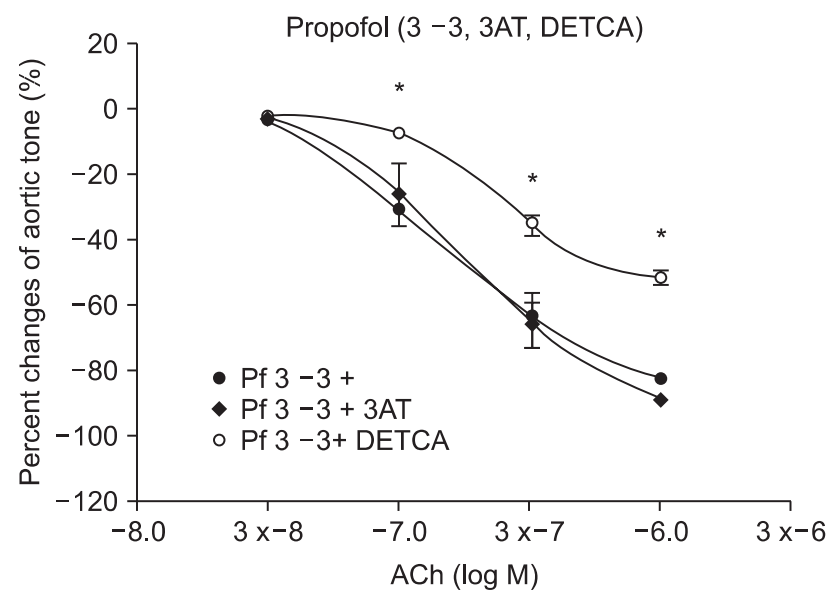

Fig. 1B. Relaxation effects of Propofol (Pf) $\left(3 \times 10^{-3} \mathrm{M}\right)$ on exposure to the reactive oxygen species (ROS) in the presence or absence of 3-amino-1,2,4-triazole (3AT), diethyldithiocarbamate (DETCA). Values are expressed as mean \pm SEM. *A P value of less than 0.001 significantly different from control group (Pf $3-3+$ ). 


\section{References}

1. Wilson JX, Gelb AW. Free radicals, antioxidants, and neurologic injury: possible relationship to cerebral protection by anesthetics. J Neurosurg Anesthesiol 2002; 14: 66-79.

2. Gumusel B, Tel BC, Demirdamar R, Sahin-Erdemli I. Reactive oxygen species-induced impairment of endothelium-dependent relaxation in rat aortic rings: protection by L-arginine. Eur J Pharmacol 1996; 306: 107-12.

3. Lamb FS, King CM, Harrell K, Burkel W, Webb RC. Free radical-mediated endothelial damage in blood vessels after electrical stimulation. Am J Physiol 1987; 252: H1041-6.

4. Schuijt MP, Tom B, de Vries R, Saxena PR, Sluiter W, van Kats JP, et al. Superoxide does not mediate the acute vasoconstrictor effects of angiotensin II: a study in human and porcine arteries. J Hypertens 2003; 21: 2335-44.

5. Rabelo LA, Cortes SF, Alvarez-Leite JI, Lemos VS. Endothelium dysfunction in LDL receptor knockout mice: a role for $\mathrm{H} 2 \mathrm{O} 2$. $\mathrm{Br} J$ Pharmacol 2003; 138: 1215-20. 